\title{
Fatores de risco para leptospirose, relacionados à atividade laboral em agricultores de arroz em uma cidade do nordeste do Brasil, 2008
}

\author{
Risk factors for leptospirosis as occupational disease in rice farming community \\ in Northeastern city in Brazil, 2008.
}

Patrícia Pereira Vasconcelos de Oliveira ${ }^{1}$, Patrícia Miyuki Ohara ${ }^{2}$, Juliano Leônidas Hoffmann ${ }^{2}$, Fernando Wilson Fernandes Silva $^{3}$, Antônia Máximo de Lima ${ }^{4}$, Maria Iracema Aguiar Patrício ${ }^{5,6}$, Maria de Lourdes Coelho Alencar Barreto ${ }^{4}$, Kátia Eliane Santos Avelar ${ }^{7}$, Jonas Lotufo Brant de Carvalho ${ }^{8}$, Wildo Navegantes de Araújo ${ }^{9}$

\begin{abstract}
1. Escola Nacional de Saúde Pública, FIOCRUZ, Rio de Janeiro, Brasil, 2. Secretaria de Vigilância em Saúde do Ministério da Saúde, Brasília, Brasil, 3. Secretaria de Saúde do Município de Várzea Alegre, Várzea Alegre, Ceará, Brasil, 4. Secretaria de Saúde do Estado do Ceará, Fortaleza, Ceará, Brasil, 5. Laboratório Central de Saúde Pública do Ceará, Brasil, 6. Doutoranda em Biotecnologia - RENORBIO, Universidade Estadual do Ceará, Brasil, 7. Laboratório de referência parra a Leptospirose, FIOCRUZ, Rio de Janeiro, Brasil, 8. Doutorando pela Universidade Estadual Paulista, UNESP, Botucatu, Brasil, 9. Universidade de Brasília, UNB - Campus Ceilândia, Brasil.
\end{abstract}

\section{Resumo}

Introdução: Em 07/2008, o município de Várzea Alegre/CE notificou 350 casos suspeitos de leptospirose em agricultores de arroz e uma investigação foi realizada para identificar os fatores de risco. Método: Um estudo de caso controle (1:2) de base populacional foi conduzido de janeiro a julho de 2008.0 caso foi definido como indivíduo que apresentou título de anticorpos para leptospirose maior ou igual a 100 pelo método da soroaglutinação microscópica e o controle foi o indivíduo saudável que não apresentou títulos de anticorpos para leptospirose selecionados aleatoriamente. Resultados: Entre os 85 casos identificados, 73(86\%) eram do sexo masculino; a mediana de idade foi de 19 anos (10-74) e mais de 60\% dos casos referiram febre, mialgia, cefaleia e calafrios. No primeiro modelo teórico, ser do sexo masculino (OR:4;IC95\%:2.3-9.4) e trabalhar na lavoura de arroz (OR:2.9;IC95\%:2.1-6.8) foram associados a doença. No segundo modelo, usar roupas curtas (OR:5.3;IC95\%:1.6-17.5) e mais de 8 horas trabalhadas na lavoura (OR:3.9;IC95\%:1.1-14.6) foram associados. Conclusões: Os principais fatores de risco identificados foram relacionados à atividade ocupacional e ao não uso de roupas de proteção individual e exposição prolongada ao ambiente de trabalho. Recomendamos o fortalecimento das políticas de saúde do trabalhador rural, aprimoramento do acesso desses agricultores às informações sobre os riscos e incentivá-los ao uso de equipamentos de proteção individual nas atividades de campo.

Palavras-chave: Leptospirose. Surtos. Área rural. Doença ocupacional. Estudo de caso controle.

\begin{abstract}
Introduction: In 2008, the city of Várzea Alegre, in Ceará State reported 350 suspected cases of leptospirosis in farmers involved in rice culture; an investigation was conducted then to identify risk factors. Method: A population-based case-control study (1:2) was performed from January to July 2008. A case was individual who presented antibody title for leptospirosis higher than or equal to 100 by microscopic agglutination method and control was healthy individuals with no antibodies for leptospirosis selected randomly. Results: Among eight five cases identified, 73(86\%) were male; median age was 29 years (10-74) and 59(69\%) were farmers. Over $60 \%$ of cases reported fever, headache, myalgia and chills. In the first theoretical model, being male (OR:4;C95\%:2.3-9.4) and working in rice crops (OR:2.9;CI95\%:2.1-6.8) were associated with leptospirosis. In the second model, using inappropriate short clothes (OR:5.3;CI95\%:1.6-17.5) and more than eight hours of daily crop work (OR:3.9;CI95\%:1.1-14.6) were associated. Conclusions: The main risk factors identified were related to the occupational activity such as not using protective equipment and prolonged exposure to the environment. We recommend the strengthening of the strategies established for a Health Workers' Policies and Agriculture Policies, by improving the workers' access to information about the risks and by encouraging the proper use of protective equipment during farm labor activities
\end{abstract}

Keywords: Leptospirosis. Disease outbreaks. Rural settings. Occupational disease. Case-control study.

\section{INTRODUÇÃO}

Apesar da melhoria das condições socioambientais e dos avanços tecnológicos, a leptospirose ainda continua sendo um problema para a saúde pública nos dias de hoje, exigindo esforços conjuntos de várias agências internacionais e lançando-se no rol das doenças negligenciadas ${ }^{(1)}$. Atualmente, cerca de 11.000 casos de leptospirose são notificados no Brasil, e destes, 28\% estão relacionados a áreas rurais. Nessas áreas, a transmissão da leptospirose ocorre de forma indireta, normalmente ligada ao processo de trabalho das pessoas que lidam com a produção de grãos e cereais ${ }^{(2)}$.

Correspondência: Patrícia Pereira Vasconcelos de Oliveira. Escola Nacional de Saúde Pública, FIOCRUZ, Rio de Janeiro, Brasil. E-mail: ppvoenf@gmail.com Conflito de interesse: Não há conflito de interesse por parte de qualquer um dos autores.

Recebido em: 10 Jul 2014; Revisado em: 14 Ago 2014; Aceito em: 2 Set 2014. 
A leptospirose como doença ocupacional acomete diferentes categorias profissionais como trabalhadores em arrozais e canaviais, minas, abatedouros e serviços de saneamento. Desde 1999, a leptospirose consta na listagem oficial de doenças originadas no processo de trabalho. No Rio Grande do Norte, epidemias rurais de leptospirose foram identificadas pela primeira vez em 1985, também entre trabalhadores das culturas de $\operatorname{arroz}^{(2)}$.

No Brasil, os custos das internações para o Sistema Único de Saúde ainda são muito altos quando comparados com o que é gasto com ações de prevenção e controle da leptospirose ${ }^{(3)}$. Em 2007, a estimativa dos custos parciais hospitalares relativos à leptospirose foi de US\$ 439.956,47. Os anos potenciais de vida perdidos são maiores na faixa etária economicamente ativa e as perdas salariais em decorrência dos óbitos causados são estimadas em US\$13.601.843,38 em salários mínimos não $\operatorname{ganhos}^{(4)}$.

Sua importância como doença ocupacional vem sendo registrada ao longo de décadas na comunidade científica ${ }^{(5,6,7)}$. Mesmo assim, medidas de controle direcionadas para agricultores e outras exposições ocupacionais não são desenvolvidas visando à sua adequação para o uso em condições adversas de temperatura e ambiente $\mathrm{e}^{(5,8-12)}$. Para a leptospirose, as medidas de prevenção e controle envolvem a comunicação e a educação à população sob risco, saneamento básico e controle de roedores(13-15). Nos países da Ásia, além das medidas citadas, são realizados tratamentos em animais e profilaxias ${ }^{(5)}$.

Em 2008, O Ministério da Saúde recebeu a notificação de um aumento de casos confirmados de leptospirose no município de Várzea Alegre, Ceará, região para a qual não havia notificação deste o agravo em anos anteriores. Após a confirmação do surto, os investigadores conduziram um estudo seccional. Os resultados demonstraram que a maioria dos casos eram de adultos jovens, do sexo masculino, agricultores e que trabalharam na lavoura do arroz em períodos de alagamento ${ }^{(16)}$.

Durante a investigação, não foram desenvolvidas análises multivariadas, que pudessem explicar o adoecimento da população do município de Várzea Alegre por leptospirose em 2008. Assim, o presente estudo teve como objetivo identificar os principais fatores associados à exposição laboral que contribuíram para o adoecimento da população, dada a especificidade do grupo mais acometido.

\section{MÉTODOS}

\section{Local de estudo:}

O município de Várzea Alegre situa-se a 467 km de Fortaleza. Com área de $836 \mathrm{~km} 2$, e possui uma população estimada em aproximadamente 38.000 habitantes. Possui cobertura da Estratégia de Saúde da Família em $100 \%$ do seu território, com 12 unidades de saúde, e um hospital com nível de atenção secundária ${ }^{(17)}$.
A base econômica do município é agrícola, sendo a cultura do arroz uma das principais cadeias produtivas, que envolve diretamente cerca de 3.000 famílias, com uma produção aproximada de 10.000 toneladas ao ano. Predomina a mão-deobra familiar, com auxílio de terceiros da própria comunidade, em sistema de mutirão, principalmente, na época da colheita do $\operatorname{arroz}^{(18)}$.

\section{Tipo de estudo}

Foi realizado um estudo analítico do tipo caso controle $e^{(1: 2)}$, de base populacional, utilizando base de dados secundária coletados no período de janeiro a julho de 2008 a partir de um questionário padronizado. A população do estudo foi composta por 281 indivíduos, selecionados durante uma investigação epidemiológica ocorrida em 2008, no município de Várzea Alegre, quando 85 foram casos confirmados de leptospirose e 196 foram controles ${ }^{(16)}$. Os controles foram selecionados por meio de sorteio, baseado no cadastro de família do Sistema de Informação da Atenção básica (SIAB), considerando a cobertura de $100 \%$ de Estratégia de Saúde da Família do município.

Considerou-se um caso confirmado de leptospirose aquele em que um indivíduo apresentasse titulação de anticorpos para leptospirose maior ou igual a 100 pelo método da soroaglutinação microscópica e um controle em que um indivíduo sadio apresentasse resultado laboratorial negativo com anticorpos para leptospirose pelo mesmo método. Para a realização do MAT foi utilizado um painel contendo cepas de referência ${ }^{(19)}$, a fim de se permitir a detecção de infecções causadas pelos sorovares mais frequentes e por sorovares incomuns ou ainda não descritos para a região.

Buscando entender os possíveis fatores de risco relacionados ao trabalho na lavoura de arroz, foi desenvolvido um segundo estudo de caso controle. Consideraram-se, para esta etapa, os casos e controles definidos no item anterior, que referiram ter trabalhado na lavoura de arroz no período do estudo. Como o número de casos que referiram trabalhar na lavoura foi maior que os de controle, decidiu-se selecionar, aleatoriamente, os casos de maneira a obter uma razão $1: 1$, permanecendo no estudo, 42 casos e 42 controles.

\section{Análise dos dados}

Todas as análises foram conduzidas no software STATA Versão 9.0. Os testes do Qui-quadrado e Exato de Fisher foram utilizados para variáveis categóricas, e para variáveis contínuas o teste de Wilcoxon. Para testar significâncias estatísticas foi considerado um nível de significância de $5 \%$ em teste bicaudal e um intervalo de confiança de $95 \%$. A Odds Ratio foi usada como medida de associação. Para identificar exposições independentemente associadas ao desfecho, realizou-se uma regressão logística, a partir do modelo teórico com todas as variáveis usando o método de eliminação por etapas para o calculo da Odds Ratio ajustada. 
$\mathrm{Na}$ análise multivariada, entraram para o primeiro modelo teórico, as exposições que obtiveram significância menor que $20 \%$ nas análises bivariadas com intervalo de confiança de $95 \%$. Para o segundo modelo, foram consideradas todas as variáveis estudadas nas análises bivariadas relacionadas ao trabalho na lavoura de arroz. A multicolinearidade entre duas ou mais variáveis foi avaliada utilizando o coeficiente de Spearman, em ambos os modelos, considerando colinear a que obteve coeficiente maior que 0,8. Por não apresentar distribuição normal, a variável idade foi normalizada calculando-se seu log. $\mathrm{Na}$ avalição do modelo foi considerada a razão de verossimilhança. Este trabalho foi aprovado pelo Comitê de Ética e Pesquisa da Escola Nacional de Saúde Pública da Fundação Oswaldo Cruz
(FIOCRUZ) por meio do parecer no 7.954 .

Dos 85 casos, 68 (80\%) foram sintomáticos. Quanto à apresentação clínica desses casos, os sinais e sintomas predominantes foram febre em 65 casos (95\%), cefaleia em 58 (85\%), mialgia em 52 (77\%), calafrio em 43 (63\%), dor na panturrilha em 37 (54\%) e náusea em 33 (48\%). Ainda foram relatados vômito e lombalgia em $44 \%$, hematúria em $43 \%$, artralgia e dor retro-orbitária em $41 \%$, dor abdominal em $40 \%$, sufusão conjuntival em $38 \%$ e diarreia em $21 \%$. Icterícia, epistaxe e hemoptise foram relatadas em menor frequência (10\%, 3\%, 3\%, respectivamente). A maior concentração de casos aconteceu entre as semanas epidemiológicas 16 a 28 (Figura 1).

Figura 1 Distribuição dos casos de leptospirose por semana epidemiológica desde o início dos sintomas (SE), Várzea Alegre/ CE, 2008. $(n=42)$

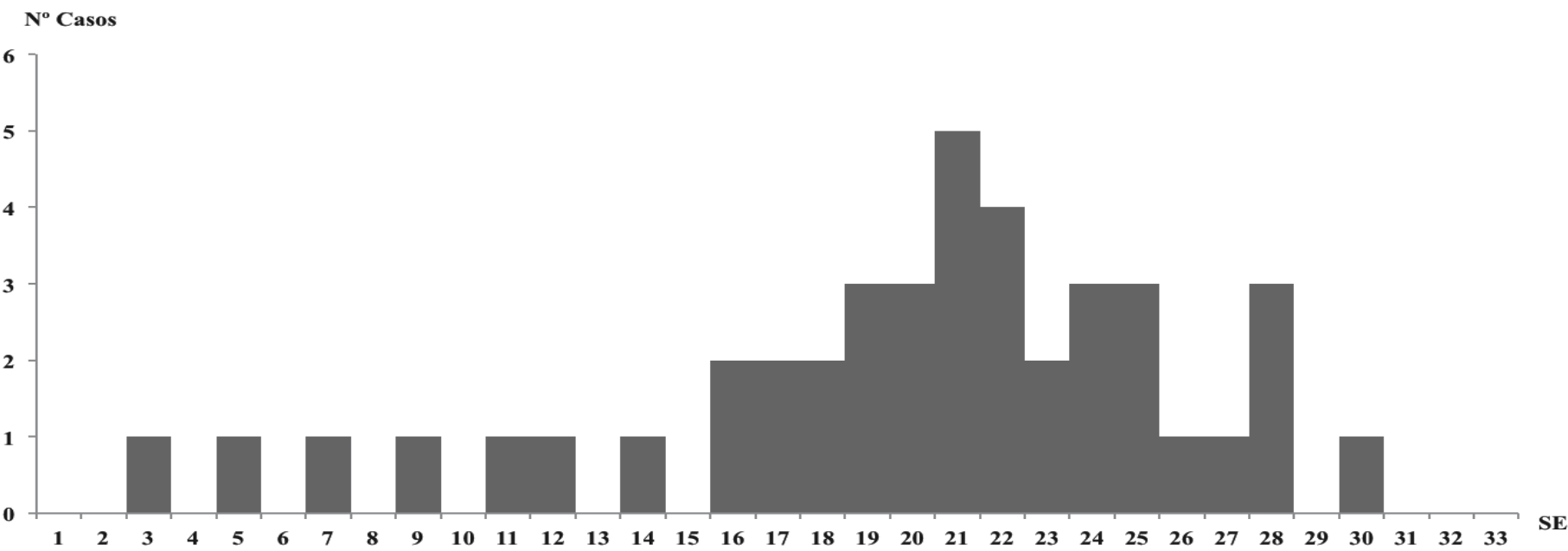

Os sorovares mais frequentes foram o Australis e Djasiman. A titulação mais frequente foi 200 , em $32 / 85$ casos. Vinte por cento (17/85) possuíram titulação 100 (Tabela 1$)$.

Dos 85 casos, $86 \%$ eram do sexo masculino e $69 \%$ eram agricultores, enquanto que, no grupo dos 196 controles, a proporção de homens foi de $45 \%$ e de agricultores foi de $34 \%$. A faixa etária predominante, tanto para casos e controles foi a de adulto jovem de 20 a 49 anos com $57 \%$ e 45\%, respectivamente.

Sessenta e um por cento dos casos e $60 \%$ dos controles possuíam escolaridade menor que oito anos de estudo. A raça/cor mais

Tabela 1. Resultado do teste de soroaglutinação microscópica dos casos de leptospirose. Várzea Alegre/CE, 2008.

\begin{tabular}{lccccccc}
\hline \multirow{2}{*}{ Relação Sorovares } & \multicolumn{7}{c}{ Título } \\
\cline { 2 - 8 } & $\mathbf{1 0 0}$ & $\mathbf{2 0 0}$ & $\mathbf{4 0 0}$ & $\mathbf{8 0 0}$ & $\mathbf{1 6 0 0}$ & $\mathbf{3 2 0 0}$ & Total \\
\hline Australis & 7 & 8 & 4 & 3 & 1 & - & 23 \\
Djasiman & 3 & 6 & 3 & 7 & 3 & 1 & 23 \\
Tarassovi & 1 & 5 & - & 1 & 2 & - & 9 \\
Cynopteri & 3 & 3 & - & - & - & - & 6 \\
Saxkoebing & - & 3 & - & 2 & - & - & 5 \\
Batavie & - & 1 & 1 & 2 & - & - & 4 \\
Hebdominales & - & 2 & 2 & - & - & - & 4 \\
Grippotyphosa & - & - & - & 1 & - & - & 1 \\
Reação entre 2 sorovares & 2 & 3 & - & 1 & 1 & 1 & 8 \\
Reação entre 3 sorovares & 1 & 1 & - & - & - & - & 2 \\
\hline Total & $\mathbf{1 7}$ & $\mathbf{3 2}$ & $\mathbf{1 0}$ & $\mathbf{1 7}$ & $\mathbf{7}$ & $\mathbf{2}$ & $\mathbf{8 5}$ \\
\hline
\end{tabular}


autorreferida, tanto pelos casos quanto pelos controles, foi a parda, com 63\% e 61\%, respectivamente. Quanto à zona de moradia, 69\% dos casos moravam na zona rural enquanto que 26 (31\%) residiam na zona urbana. Para os controles, a proporção de residência em zona rural e urbana foi de $50 \%$. A mediana da renda familiar foi $\mathrm{R} \$ 200,00$ para os casos e $\mathrm{R} \$ 415,00$ para os controles. Outros aspectos sociodemográficos dos grupos estão descritos na tabela 2 .
Nas análises bivariadas das características gerais, os casos tiveram mais chance de ser do sexo masculino $(\mathrm{OR}=7,3$; IC95\% 3,6-15,5), de serem agricultores (OR=4,4; IC95\% 2,4$7,9)$, de residirem em área rural (OR=2,2; IC95\% 1,3-4,0), de se banharem em algum tipo de coleção hídrica com água parada (OR=2,2; IC95\% 1,2-4,0) e de consumirem bebida alcoólica (OR=2,9; IC95\% 1,5-5,1) estatisticamente significativos quando comparados aos controles. A chance dos casos terem

Tabela 2. Características sociodemográficas dos casos e controles. Várzea Alegre/CE. 2008.

no(\%)

\begin{tabular}{lll}
\cline { 2 - 3 } Características & Casos $(\mathrm{n}=85)$ & Controles $(\mathrm{n}=196)$
\end{tabular}

\section{Sexo}

Masculino

$89(45)$

Feminino

12(14)

107(56)

\section{Faixa Etária}

10 a 19 anos

20 a 49 anos

Acima 50 anos

Escolaridade

$<8$ anos

$\geq 8$ anos

\section{Ocupação}

Agricultor

Estudante

Dona de casa

Outros*

\section{Raça/Cor}

Branca

Negra

Parda

Amarela

Indígena

Não sabe

\section{Zona de residência}

Rural

Urbana

Incinera

$$
\begin{aligned}
& 40(48) \\
& 26(31) \\
& 16(19) \\
& 02(02)
\end{aligned}
$$$$
108 \text { (55) }
$$

\section{Mediana (Intervalo)}

200,00 (IIQ*: 101,00 a 500,00) 01 (IIQ: 01 a 02)
415,00 (IIQ*: 122,00 a 615,00) 01 (IIQ: 01 a 04) 
trabalhado na lavoura de arroz foi seis vezes maior em relação aos controles (OR=6,0; IC95\% 3,2-10,4) (Tabela 3).

Entre as exposições relacionadas diretamente ao trabalho na lavoura, foi significativamente associada ao adoecimento por leptospirose; a variável independente "uso de roupas curtas inapropriadas em qualquer uma das fases plantio, alagamento ou colheita" (OR=2,9; IC95\% 1,1-7,9) (Tabela 3). As demais exposições: trabalho com níveis baixos de água $(15 \mathrm{~cm})$; trabalho na fase do plantio, do alagamento ou da colheita; relato da presença de roedores na lavoura durante a jornada de trabalho; trabalho com algum tipo de ferimento no corpo; jornada de mais de oito horas na lavoura; dois a quatro dias de trabalho na lavoura e hábito de armazenar os grãos colhidos na própria residência; apresentaram associação ao desfecho, porém não obtiveram significância estatística nas análises bivariadas (Tabela 4).

Tabela 3. Exposições associadas ao adoecimento por leptospirose. Análises bi e multivariada. Várzea Alegre/CE, 2008.

\begin{tabular}{|c|c|c|c|c|c|c|c|c|}
\hline \multirow[b]{2}{*}{$\begin{array}{l}\text { Exposições relacionadas às condições so- } \\
\text { ciais e hábitos }\end{array}$} & \multicolumn{3}{|c|}{ n (\%) } & \multicolumn{3}{|c|}{ Bivariada } & \multicolumn{2}{|c|}{ Multivariada } \\
\hline & $\begin{array}{l}\text { Casos } \\
(n=85)\end{array}$ & $\begin{array}{l}\text { Controles } \\
(n=196)\end{array}$ & OR & IC95\% & p-valor & $\mathrm{aOR}^{\mathfrak{d}}$ & IC95\% & p-valor \\
\hline Sexo masculino & $73(86)$ & $89(45)$ & 7,3 & $3,6-15,5$ & $<0,001$ & 4,0 & $2,3-9,4$ & $<0,001$ \\
\hline Idade entre 50 e 64 anos $^{E}$ & $07(08)$ & 37 (19) & 0,4 & $0,1-0,9$ & 0,031 & 0,4 & $0,1-1,01$ & 0,052 \\
\hline Agricultor & $59(69)$ & $99(50)$ & 4,4 & $2,4-7,9$ & $<0,001$ & 1,8 & $0,9-3,7$ & 0,092 \\
\hline Escolaridade $<8$ anos & $52(61)$ & $118(60)$ & 1,0 & $0,6-1,8$ & 0,878 & - & - & - \\
\hline Morador da zona rural & $59(69)$ & $99(50)$ & 2,2 & $1,3-4,0$ & 0,003 & - & - & - \\
\hline Residência próxima a coleções híbridas & $43(51)$ & 77 (39) & 1,6 & $0,9-2,7$ & 0,078 & - & - & - \\
\hline Consumo de bebidas alcoólicas & $35(41)$ & $39(20)$ & 2,9 & $1,5-5,1$ & $<0,001$ & - & - & - \\
\hline Banho em coleções híbridas & $34(40)$ & $45(23)$ & 2,2 & $1,2-4,0$ & 0,003 & - & - & - \\
\hline $\begin{array}{l}\text { Costuma reunir entulho ao redor da } \\
\text { residência }\end{array}$ & $41(49)$ & 77 (39) & 1,5 & $0,8-2,5$ & 0,148 & - & - & - \\
\hline Contato com animais domésticos & $57(67)$ & $117(60)$ & 1,4 & $0,8-2,4$ & 0,242 & - & - & - \\
\hline Trabalho na lavoura de arroz & $52(62)$ & $42(21)$ & 6,0 & $3,2-10,4$ & $<0,001$ & 2,9 & $2,1-6,8$ & 0,003 \\
\hline
\end{tabular}

థ Ajustadas pelas demais variáveis do modelo

$£$ Referência 10 a 14 anos

Tabela 4. Exposições relacionadas ao trabalho na lavoura associadas ao adoecimento por leptospirose. Análises bi e multivariada. Várzea Alegre/CE, 2008.

\begin{tabular}{|c|c|c|c|c|c|c|c|c|}
\hline \multirow{2}{*}{$\begin{array}{l}\text { Exposições relacionadas ao trabalho na } \\
\text { lavoura }\end{array}$} & \multicolumn{3}{|c|}{ n (\%) } & \multicolumn{3}{|c|}{ Bivariada } & \multicolumn{2}{|c|}{ Multivariada } \\
\hline & $\begin{array}{l}\text { Casos } \\
(n=46)\end{array}$ & $\begin{array}{l}\text { Controles } \\
(n=46)\end{array}$ & OR & IC95\% & p-valor & $\mathbf{a O R}^{\mathbb{q}}$ & IC95\% & p-valor \\
\hline Trabalho com níveis baixos de água $(15 \mathrm{~cm})$ & $23(55)$ & $27(64)$ & 0,7 & $0,3-1,8$ & 0,373 & 0,3 & $0,9-1,0$ & 0,055 \\
\hline Trabalho na fase do plantio & $40(98)$ & $38(90)$ & 4,2 & $0,4-212,4$ & 0,175 & 3,5 & $0,2-51,1$ & 0,366 \\
\hline Trabalho na fase do alongamento & $35(83)$ & $36(86)$ & 0,8 & $0,2-3,2$ & 0,762 & - & - & - \\
\hline Trabalho na fase da colheita & $48(92)$ & $36(86)$ & 1,8 & $0,3-12,4$ & 0,435 & - & - & - \\
\hline Uso de roupas curtas e chinelos ou descalço & $25(59)$ & $14(33)$ & 2,9 & $1,1-7,9$ & 0,016 & 5,3 & $1,6-17,5$ & 0,006 \\
\hline Relato da presença de roedores na lavoura & $27(64)$ & $23(55)$ & 1,5 & $0,6-3,9$ & 0,373 & - & - & - \\
\hline $\begin{array}{l}\text { Trabalho com algum tipo de ferimento no } \\
\text { corpo }\end{array}$ & $05(12)$ & $04(10)$ & 1,2 & $0,2-6,6$ & $0,782^{*}$ & - & - & - \\
\hline Mais de 8 horas de trabalho na lavoura ${ }^{£}$ & $14(33)$ & $08(19)$ & 2,1 & $0,7-6,7$ & 0,272 & 3,9 & $1,1-14,6$ & 0,042 \\
\hline 2 a 4 dias trabalhando na lavoura\# & $42(81)$ & $32(76)$ & 1,1 & $0,4-3,6$ & 0,794 & - & - & - \\
\hline $\begin{array}{l}\text { Armazenamento de grãos colhidos na } \\
\text { residência. }\end{array}$ & $29(76)$ & $23(66)$ & 1,7 & $0,5-5,3$ & 0,317 & 2,4 & $0,8-7,9$ & 0,135 \\
\hline
\end{tabular}

* Exato de Fisher

c Ajustadas pelas demais variáveis do modelo

f Referência: Menos de 8 horas de trabalho na lavoura

\# Referência: 6 a 8 dias de trabalho na lavoura 
As variáveis utilizadas no primeiro modelo teórico foram sexo, idade, trabalho na lavoura de arroz, consumo de bebidas alcoólicas, hábito de banhar-se em coleções hídricas, proximidade da residência de coleções hídricas e hábito de reunir entulhos ao redor da residência. Nenhuma variável proposta para o modelo foi retirada devido às características colineares.

Neste primeiro modelo permaneceram independentemente associadas ao adoecimento por leptospirose: ser do sexo masculino ( $a O R=4,0 ; I C 95 \% 2,3-9,4)$ e ter trabalhado na lavoura de arroz (aOR=2,9; 2,1-6,8). A faixa etária de referência (10 a 14 anos) obteve uma estimativa de risco $60 \%$ menor quando comparada à faixa etária de 50 a 64 anos, porém esta associação não apresentou significância estatística. (Tabela 3).

Foram usadas no segundo modelo todas as variáveis relacionadas ao trabalho na lavoura, independente da significância estatística nas análises bivariadas. A variável "trabalho na fase da colheita" não foi inserida no modelo por apresentar colinearidade com as demais variáveis. Sendo assim, o modelo foi composto pelas variáveis trabalho na lavoura com níveis baixos de água $(15 \mathrm{~cm})$, trabalho na fase do plantio, uso de roupas curtas sem botas, mais de oito horas de trabalho na lavoura e armazenamento de grãos colhidos na residência, medida preditora indireta para a presença de roedores na residência.

O trabalho realizado com o uso de roupas curtas e jornada de mais de oito horas de trabalho na lavoura foram relacionadas, significativamente, o desfecho, ou seja, os casos tiveram uma chance cinco e quatro vezes maior de terem usado roupas curtas e trabalhado por mais de oito horas na lavoura, respectivamente (IC95\% 1,6-17,5 e IC95\% 1,1-14,6) (Tabela 4), conforme demonstrado na figura 2.

Figura 2. Exposições vivenciadas pelos agricultores de Várzea Alegre/CE durante o trabalho na lavoura de arroz, 2008.
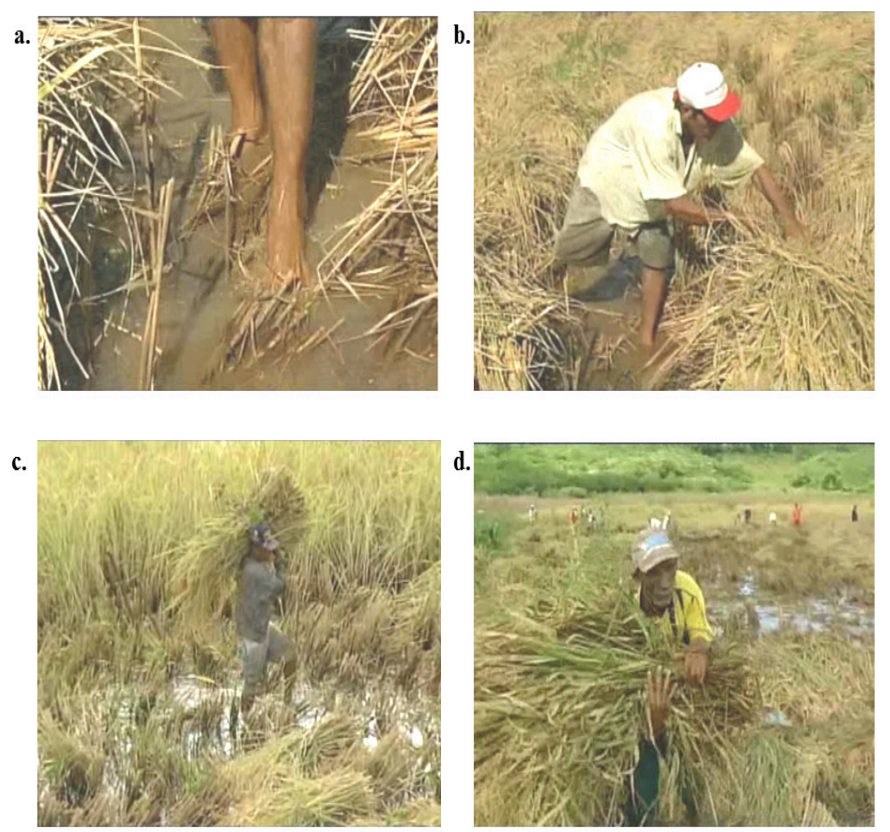

\section{Discussão}

Os principais fatores de risco encontrados neste estudo estiveram relacionados à atividade ocupacional desenvolvida por grande parte das pessoas acometidas por leptospirose. Os homens que trabalharam na lavoura de arroz utilizando vestimentas curtas as quais favoreciam uma maior exposição do corpo ao ambiente contaminado e acima de oito horas de trabalho demonstraram ter uma maior probabilidade de adoecimento em Várzea Alegre, no ano de 2008.

Assim como em estudos realizados na Nicarágua, Peru e em áreas urbanas brasileiras, o sexo masculino permaneceu associada ao adoecimento de forma independente ${ }^{(11-16 ; 20-23)}$. Tal observação evidencia uma maior exposição da população masculina ao ambiente de risco. Muitas vezes, essa exposição está associada à atividade ocupacional, considerada predominantemente masculina, como é o caso de trabalhadores de abatedouros, frigoríficos, agricultura, e em esgotos. Lomar et al. (2005) (24) relatam que, quando ambos os sexos estão expostos às fontes de contágio, não existe diferença de susceptibilidade; porém, uma outra hipótese para explicar a predominância do sexo masculino podem ser relacionados a fatores hormonais, o que incita discussões e desafios ao Sistema Único de Saúde na perspectiva de se discutirem estratégias de enfrentamento da leptospirose com maior atenção para as especificidades de gênero .

Trevejo et. al. (1998) $)^{(14)}$ encontraram uma incidência significativamente maior na faixa etária de 1 a 14 anos, comparada com outros grupos de idade, em 1995, durante uma epidemia de febre hemorrágica em uma área rural da Nicarágua, após período de fortes chuvas. Porém, em nosso estudo, observou-se que a faixa etária de 50 a 64 anos, apresentou um risco menor de adoecimento quando comparado à faixa de 10 a 14 anos. Isso pode ter ocorrido em Várzea Alegre pelo fato de a força de trabalho ser composta predominantemente por jovens, em relação aos mais velhos.

Embora a leptospirose ainda se encontre relacionada com baixas condições socioeconômicas como: baixa escolaridade, pobreza, ausência de saneamento básico ${ }^{(25-27)}$, em nosso estudo, a associação com a escolaridade não foi encontrada, talvez por essas características se apresentarem homogêneas na população desta cidade, especificamente. Entretanto, a ocupação de agricultor apresentou uma forte associação.

A exposição ocupacional entre agricultores também foi encontrada em outros países como Taiwan, Sri Lanka, Trinidad, Índia e Havaí(10,13,22,28-30). Na Europa, em 2007, um surto de leptospirose foi identificado entre trabalhadores rurais envolvidos com o cultivo de morangos ${ }^{(31)}$. No Brasil, apesar das características ocupacionais não serem rurais, trabalhadores do serviço de saneamento ambiental na região sul do país também foram acometidos pela doença ${ }^{(32)}$. Outro estudo desenvolvido no Paraná identificou alta prevalência de leptospirose em trabalhadores de matadouros ${ }^{(33)}$. No Rio Grande do Norte, um estudo de soro prevalência identificou que $98 \%$ dos indivíduos 
trabalhavam em atividades agrícolas ${ }^{(2)}$.

Em Várzea Alegre, o trabalho na lavoura do arroz foi a principal atividade relatada pelos indivíduos e foi associada de maneira independente com o adoecimento. Na Tailândia, também foi observada a associação desta atividade com maior risco de contrair leptospirose, o que é explicado pelo uso de terrenos alagados para o cultivo ${ }^{(34)}$. Na Índia, 68\% dos agricultores de arroz foram soro reagentes para a leptospirose ${ }^{(7)}$.

Entre as exposições relacionadas ao trabalho na lavoura, o uso de roupas curtas, ou o não uso de sapatos no trabalho da lavoura por mais de oito horas, aumentaram o risco de contrair a doença. Na Índia, foi encontrado um risco quase três vezes maior de contrair a doença quando não foram usados calçados na lavoura ${ }^{(29)}$. Na Tailândia, a associação foi cerca de sete vezes maior, porém não apresentou significância estatística ${ }^{(34)}$. No mesmo país, em 1999, um estudo realizado com pessoas que limpavam barragens, lagos e canais, demonstrou que o uso de calças compridas ou camisas longas ou até mesmo qualquer tipo de calçado apropriado para a água tiveram um efeito protetor contra a leptospirose ${ }^{(12)}$.

As feridas também são relatadas como fatores de risco importante tanto nas exposições ocupacionais, quanto em atividades de lazer ${ }^{(12,28-29,35)}$. O mesmo estudo da Tailândia demonstrou que as pessoas com leptospirose que trabalhavam com atividades de limpeza de barragens, lagoas e canais tiveram uma chance três vezes maior de ter uma média de duas ou mais feridas no momento do trabalho quando comparados aos que não adoeceram ${ }^{(15)}$. Em nosso estudo, tal associação não foi encontrada; contudo, não descartamos a importância dos pequenos ferimentos como porta de entrada da leptospira, uma vez que o cultivo do arroz pode levar a pequenos ferimentos não valorizados no cotidiano dos agricultores.

Apesar de não apresentar associação direta com a doença, a visualização de ratos no ambiente de trabalho pode ser um preditor importante para a presença/densidade do reservatório silvestre no ambiente. Essa medida indireta também foi avaliada em outros estudos, porém não foram encontradas associações ${ }^{(21,36)}$. Porém, apesar do estudo ter sido feito em uma área urbana, onde os reservatórios implicados são diferentes dos observados em nosso estudo, Lima (2010) identificou uma associação entre avistar ratos no ambiente peridomiciliar e ter diagnóstico confirmado para leptospirose. No entanto, em um ambiente rural, outros animais podem ter alguma relação com a contaminação do ambiente e, consequentemente, com a transmissão indireta ao homem. Sendo assim, em situações como essa, deveriam ser investigados tanto os casos humanos quanto os animais que estavam presentes no momento do surto, pois uma importante vigilância de casos humanos e animais pode ser mais efetiva e oportuna para a prevenção e controle da leptospirose ${ }^{(5)}$.

No Brasil, não há uma decisão explícita do Programa Nacional de Controle da Leptospirose sobre a quimioprofilaxia como medida preventiva para reduzir o risco de infecção em populações de lavradores ou em pessoas que possam adoecer por relação com seu trabalho ${ }^{(38)}$. No tocante à nossa população de estudo, é necessário avaliar se essa seria uma estratégia eficaz, pois existem estudos que demostram que, embora a quimioprofilaxia seja uma medida de prevenção importante para grupos de viajantes a áreas de risco, seu uso em grandes populações talvez venha a ser impraticável ${ }^{(13)}$.

Ainda não há disponível, no mercado brasileiro, uma vacina para seres humanos. Medidas de prevenção para exposições ocupacionais devem recomendar o uso de luvas, botas e vestimentas à prova d' água, além do conhecimento a respeito dos riscos que os ferimentos na pele representam ${ }^{(12)}$. Porém, existem controvérsias quanto aos tipos de Equipamento de Proteção Individual (EPI) que devem ser usados pelo trabalhador rural, pois, mesmo que esses equipamentos estejam voltados para os riscos relacionados ao manuseio de substâncias tóxicas, eles podem não ser adequados às situações climáticas às quais essa população está exposta, bem como a seu elevado custo de aquisição(8).

A baixa titulação considerada no estudo pode ter causado a inclusão de casos não relacionados ao surto de 2008, pois estudos mostram que titulações abaixo de 100 evidenciam contatos prévios com a doença ${ }^{(11,20)}$. Em contrapartida, tal fato permitiu aumentar a sensibilidade da definição de caso, visto que, até o ano de 2008, o município de Várzea Alegre não apresentava casos notificados de leptospirose no sistema de informação. Esse fato sugere que, em Várzea Alegre, poderiam ter ocorrido casos de leptospirose, em períodos anteriores ao surto, que não foram identificados pela Vigilância Epidemiológica e, consequentemente, não notificados ao Sinan.

Também não foi realizado o pareamento das amostras para a realização do MAT. Isto pode ter ocorrido por se tratar de uma situação de surto que exige uma resposta rápida do sistema, pelas dificuldades logísticas em obter duas amostras em localidades predominantemente rurais e pela não oportunidade da investigação, que se iniciou apenas seis meses após o aparecimento dos casos.

Além do mais, apesar de o estudo ter sido de base populacional, da não oportunidade da investigação, do ponto de corte baixo para a titulação e da não realização de amostras pareadas de MAT, não foi possível estimar a real magnitude do surto por tratar-se de casos prevalentes, e não incidentes, que poderiam ter sido afetados em anos anteriores devido à manutenção de títulos ser prolongada por vários anos ${ }^{(19)}$. Entre as possíveis causas de não haver registros de casos de leptospirose em anos anteriores, estão a fragilidade do sistema de vigilância em não ser capaz de detectar surtos e de haver subnotificação em relação aos casos leves da doença, ou seja, a classificação errônea para outras doenças devido a uma gama de diagnósticos diferenciais possíveis ${ }^{(3,5,19,20,26)}$.

Observou-se, ainda, que não foi realizada, nesse surto, a investigação ambiental ou sorologia animal, o que implicou no desconhecimento do reservatório envolvido na transmissão 
humana, algo importante para ser verificado em áreas rurais, devido à escassez de informações referentes ao Brasil.

Mediante os resultados apresentados, é imprescindível o fortalecimento do Programa Nacional de Controle da Leptospirose e, de fato, a implantação do Programa de Saúde do Trabalhador e da Trabalhadora, no sentido de interiorizar as ações para que os municípios pequenos tenham estrutura de diagnóstico diferencial, com abordagem sindrômica e tratamento oportuno, bem como atividades de educação e de alerta aos trabalhadores expostos às atividades de risco e de esclarecimentos sobre seus direitos.

Sendo assim, consideramos importante o fortalecimento das ações de saúde do trabalhador alinhadas com a atenção básica, no sentido de detectar precocemente os riscos, tratar oportunamente os acometidos e manter a vigilância em alerta nas fases de maior exposição, conforme preconizado pela Política Nacional de Saúde do Trabalhador e da Trabalhadora ${ }^{(38)}$.

\section{REFERÊNCIAS BIBLIOGRÁFICAS}

1.Cachay ER, Vinetz JM. A global research agenda for leptospirosis. J Postgrad Med. 2005 Jul-Sep;51(3):174-8. PubMed PMID: 16333188.

2.Lacerda HG, Monteiro GR, Oliveira CCG, Suassuna FB, Queiroz JW, Barbosa JDA et al. Leptospirosis in a subsistence farming community in Brazil. Trans $R$ Soc Trop Med Hyg. 2008 Dec; 102(12): 1233-1238. PubMed PMID: 18599101.

3.Souza VMM, Brant JL, Arsky MLS, Araújo WN. Avaliação do sistema nacional de vigilância epidemiológica da leptospirose - Brasil, 2007. Cad. Saúde Colet. 2010 jan-mar; 18(1): 95-105.

4.Souza VMM, Simões MLN, Castro APB, Araujo WN. Anos potenciais de vida perdidos e custos hospitalares da leptospirose no Brasil. Rev Saúde Pública. 2011 dez; 45(6). doi: http://dx.doi.org/10.1590/S0034-89102011005000070.

5.Waitkins SA. Leptospirosis as an occupational disease. Br J Ind Med. 1986 Nov; 43(11): 721-5. PubMed Central PMCID: PMC 1007745.

6.Katz RA AV, Effler PV, Middleton CR, Sasaki DM. Leptospirosis in Hawaii, 19741998: epidemiologic analysis of 353 laboratory-confirmed cases. Am J Trop Med Hyg. 2002 Jan; 66(1):61-70. PubMed PMID: 12135270.

7.Natarajaseenivasan K, Boopalan M, Selvanayaki K, Suresh SR, Ratnam S. Leptospirosis among rice mill workers of Salem, South India. Jpn J Infect Dis. 2002 Oct;55(5):170-3. PubMed PMID: 12501258.

8.Almeida RACS, Veiga MM. Processo de trabalho rural e EPI's: discussão sobre termorregulação corporal. Revista P\&D em Engenharia de Produção. 2010; 8(2):29-39.

9.Johnson MAS, Smith H, Joseph P, Gilman RH, Bautista CT, Campos KJ, et al. Environmental exposure and leptospirosis, Peru. Emerg Infect Dis. 2004 Jun;10(6): 1016-22. PubMed PMID: 15207052.

10.Gamage CD, Amarasekera J, Palihawadana P, Samaraweera S, Mendis D, Janakan N, et al. Analysis of Hospital-Based Sentinel Surveillance Data on Leptospirosis in Sri Lanka, 2005-2008. Jpn J Infect Dis. 2012; 65(2):157-61. PubMed PMID: 22446124.

11.Reis RB, Ribeiro GS, Felzemburgh RDM, Santana FS, Mohr S, Melendez AXTO, et al. Impact of Environment and Social Gradient on Leptospira Infection in Urban Slums. PLoS Negl Trop Dis. 2008 Apr 23; 2(4): e228. doi: 10.1371/journal. pntd.0000228. PubMed Central PMCID: PMC 2292260.

12.Phraisuwan $P$, Whitney EAS, Tharmaphornpilas $P$, Guharat $S$, Thongkamsamut $S$, Aresagig S, et al. Leptospirosis: skin wounds and control strategies, Thailand, 1999. Emerg Infect Dis. 2002 Dec; 8(12):1455-9. PubMed PMID: 12498663.

13.Zavitsanou Z, Babatsikou F. Leptospirosis: epidemiology and preventive measures. Health Science Journal. 2008; 2(2):75-82.

14.Trevejo RT, Rigau-Pérez JG, Ashford DA, McClure EM, Jarquín-González C, Amador JJ, et al. Epidemic leptospirosis associated with pulmonary hemorrhageNicaragua, 1995. JID. 1998;178(5):1457-63. PubMed PMID: 9780268.

15.Levett PN. Leptospirosis. Clin Microbiol Rev 2001 Apr;14(2): 296-326. doi:

\subsection{8/CMR.14.2.296-326.2001}

16.Oliveira PPV. Fatores de risco para leptospirose como doença ocupacional em surto no interior do Ceará: estudo de caso controle [Dissertação]. Rio de Janeiro: FIOCRUZ; 2012.

17.Várzea Alegre [Internet]. Wikipédia, a enciclopédia livre; [atualizada em 2014 ago. 25; citado em: 2011 mar. 12]. Disponível em: <http://pt.wikipedia. org/wiki/Várzea_Alegre >

18.Prefeitura de Várzea Alegre [Internet]. Prefeitura de Várzea Alegre; [citado em: 2011 mar. 08]. Disponível em: < Em: <http://www.varzeaalegre.ce.gov.br/>

19.World Health Organization. Human Leptospirosis: Guidance for Diagnosis, Surveillance and Control. Geneva, Switzerland: WHO, 2003, 109p.

20.Dias JP, Teixeira MG, Costa MCN, Mendes CMC, Guimarães P, Reis MG, et al. Factors associated with Leptospira $\mathrm{sp}$ infection in a large urban center in northeastern Brazil. Ver Soc Bras Med Trop. 2007 Sep-Oct;40(5):499-504. PubMed PMID: 17992402.

21.Kawaguchi L, Sengkeopraseuth B, Tsuyuoka R, Koizumi N, Akashi H, Vongphrachanh $\mathrm{P}$, et al. Seroprevalence of leptospirosis and risk factor analysis in flood-prone rural areas in Lao PDR. A J Trop Med Hyg. 2008;78(6):957-61. PubMed PMID: 18541776.

22.Everard CO, Fraser-Chanpong GM, Hayes R, Bhagwandin LJ, Butcher LV. A survey of leptospirosis in febrile patients mainly from hospitals and clinics in Trinidad. Trans R Soc Trop Med Hyg. 1982 Jun;76(4):487-92. PubMed PMID: 6926766.

23.Tan DS. Leptospirosis in rural West Malaysia. Med J Malaya. 1970 Jun;24(4):261-6. PubMed PMID: 4248346.

24.Lomar, AV; Diament D; Brito T; Veronesi, R. Leptospiroses In: Veronesi. Tratado de infectologia. 3.ed. São Paulo: Atheneu; 2005. p 1239-1255.

25. Ko Al, Galvao Reis M, Ribeiro Dourado CM, Johnson Jr WD, Riley LW. Urban epidemic of severe leptospirosis in Brazil. Salvador Leptospirosis Study Group. Lancet. 1999 Sep 4;354(9181): 820-5. PubMed PMID: 10485724.

26.Ashford DA, Kaiser RM, Spiegel RA, Perkins BA, Weyant RS, Bragg SL et al. Asymptomatic infection and risk factores for leptospirosis in Nicaragua. Am J Trop Med Hyg. 2000 Nov-Dec; 63(5-6). 249-54. PubMed PMID: 11421372.

27.Costa E, Costa YA, Lopes AA, Sacramento E, Bina JC. Formas graves de leptospirose: aspectos clínicos, demográficos e ambientais. Rev Soc Bras Med Trop. 2001 maio-jun; 34(3):261-7.

28.Sasaki DM, Pang L, Minette HP, Wakida CK, Fujimoto WJ, Manea SJ, et al. Active surveillance and risk factors for leptospirosis in Hawaii. A J Trop Med Hyg. 1993 Jan;48(1):35-43. PubMed PMID: 8427386.

29.Sugunan AP, Vijayachari P, Sharma S, Roy S, Manickam P, Natarajaseenivasan $\mathrm{K}$, et al. Risk factors associated with leptospirosis during an outbreak in Middle Andaman, India. Indian J Med Res. 2009 Jul;130(1):67-73. PubMed PMID: 19700804. 
30.Tsai CC, Fresh JW. Serological study of leptospirosis among farmers and farm helpers in central Taiwan. Zhonghua Min Guo Wei Sheng Wu Xue Za Zhi.. 1974 Jul;7(4):176-7. PubMed PMID: 4480434.

31.Desai S, Van Treeck U, Lierz M, Espelage W, Zota L, Czerwinski M, et al. Resurgence of field fever in a temperate country: an epidemic of leptospirosis among seasonal strawberry harvesters in Germany in 2007. Clin Infect Dis. 2009 Mar; 48(6):p. 691-7. doi: 10.1086/597036. PubMed PMID: 19193108.

32.Almeida LP, Martins LFS, Brod CS, Germano PML. Levantamento soroepidemiológico de leptospirose em trabalhadores do serviço de saneamento ambiental em localidade urbana da região. sul do Brasil. Rev. Saúde Pública. 1994 fev; 28(1): p.76-81. doi: http://dx.doi.org/10.1590/S003489101994000100009.

33.Gonçalves DD, Teles PS, Reis CR, Lopes FMR, Freire RL, Navarro IT, et al. Seroepidemiology and occupational and environmental variables for leptospirosis, brucellosis and toxoplasmosis in slaughterhouse workers in the Paraná State, Brazil. Rev. Inst. Med. Trop. São. Paulo, 2006 May-Jun; 48(3): p.135-140. PubMed PMID: 16847502.

34.Tangkanakul W, Tharmaphornpil P, Plikaytis BD, Bragg S, Poonsuksombat D,
Choomkasien $\mathrm{P}$, et al. Risk factors associated with leptospirosis in northeastern Thailand, 1998. A J Trop Med Hyg. 2000 Sep-Oct;63(3):204-8. PubMed PMID: 11388516.

35.Morgan J, Bornstein SL, Karpati AM, Bruce M, Bolin CA, Austin CC, et al. Outbreak of leptospirosis among triathlon participants and community residents in Springfield, Illinois, 1998. Clin Infect Dis. 2002 Jun;34(12):1593. PubMed PMID: 12032894

36. Reis RB, Ribeiro GS, Felzemburgh RDM, Santana FS, Mohr S, Melendez AXTO, et al. Impact of Environment and Social Gradient on Leptospira Infection in Urban Slums. PLoS Negl Trop Dis. 2008 Apr 23;2(4):e228. doi: 10.1371/journal. pntd.0000228. PubMed PMID: 18431445

37.Lima HCAV. Incidência de fatores de risco associados às diferentes formas clínicas da leptospirose: um estudo de vigilância de base populacional em uma comunidade urbana de Salvador-Bahia. [Dissertação]. Salvador: Fundação Oswaldo Cruz; 2010.

38.BRASIL. Ministério da Saúde Portaria no 1.823, de 23 de agosto de 2012. Institui a Política Nacional de Saúde do Trabalhador e da Trabalhadora. Diário Oficial da República Federativa do Brasil. 2012 ago. 24; Seção .1 p. 46-51.

Como citar este artigo/How to cite this article:

Oliveira PPV, Ohara MP, Hoffmann JL, Silva FWF, Lima AML, Patrício MIA, Barreto MLCA, Avelar KES, Carvalho JLB, Araújo WN. Fatores de risco para leptospirose, relacionados à atividade laboral em agricultores de arroz em uma cidade do nordeste do Brasil, 2008 . J Health Biol Sci. 2014 Jul-Set; 2 (3):99-107. 